Accepted by Astrophysical Journal

\title{
Polarimetry of Compact Symmetric Objects
}

\author{
N.E. Gugliucci ${ }^{1,3}$, G.B. Taylor ${ }^{2,3}$, A.B. Peck ${ }^{4}$, and M. Giroletti ${ }^{5,6}$ \\ neg9j@virginia.edu; gbtaylor@unm.edu; apeck@cfa.harvard.edu; \\ giroletti@ira.inaf .it
}

\begin{abstract}
We present multi-frequency VLBA observations of two polarized Compact Symmetric Objects (CSOs), J0000+4054 and J1826+1831, and a polarized CSO candidate, J1915+6548. Using the wavelength-squared dependence of Faraday rotation, we obtained rotation measures $(\mathrm{RMs})$ of $-180 \pm 10 \mathrm{rad} \mathrm{m}^{-2}$ and $1540 \pm 7 \mathrm{rad} \mathrm{m}^{-2}$ for the latter two sources. These are lower than what is expected of CSOs (several $1000 \mathrm{rad} \mathrm{m}^{-2}$ ) and, depending on the path length of the Faraday screens, require magnetic fields from 0.03 to $6 \mu \mathrm{G}$. These CSOs may be more heavily affected by Doppler boosting than their unpolarized counterparts, suggesting that a jet-axis orientation more inclined towards the line of sight is necessary to detect any polarization. This allows for low RMs if the polarized components are oriented away from the depolarizing circumnuclear torus. These observations also add a fourth epoch to the proper motion studies of J0000+4054 and J1826+1831, constraining their kinematic age estimates to $>610$ yrs and $2600 \pm 490$ yrs, respectively. The morphology, spectrum, and component motions
\end{abstract}

\footnotetext{
${ }^{1}$ Department of Astronomy, P.O. Box 400325, University of Virginia, Charlottesville, VA 22904; neg9j@virginia.edu

${ }^{2}$ Department of Physics and Astronomy, University of New Mexico, Albuquerque, NM 87131; gbtaylor@unm.edu

${ }^{3}$ National Radio Astronomy Observatory, P.O. Box O, Socorro, NM 87801

${ }^{4}$ Harvard-Smithsonian Center for Astrophysics, SAO/SMA Project, 645 North A'ohoku Place, Hilo, HI 96720; apeck@cfa.harvard.edu

${ }^{5}$ INAF Istituto di Radioastronomia, via Gobetti 101, 40129 Bologna, Italy; giroletti@ira.inaf.it

${ }^{6}$ Dipartimento di Astronomia dell'Università di Bologna, via Ranzani 1, 40127 Bologna, Italy
} 
of J1915+6548 are discussed in light of its new classification as a CSO candidate, and its angle to the line of sight $\left(\sim 50^{\circ}\right)$ is determined from relativistic beaming arguments.

Subject headings: galaxies: active - galaxies: ISM - galaxies: jets - galaxies: nuclei - radio continuum: galaxies

\section{Introduction}

Compact symmetric objects (CSOs) are a class of active galactic nuclei (AGN) that have significant visible jet or hotspot activity on either side of the central engine (Conway et al. 1994; Wilkinson et al. 1994). These are typically $<1 \mathrm{kpc}$ in size because they are young objects ( $\leq 3000$ yr; Polatidis \& Conway 2003; Gugliucci et al. 2005). In terms of the unified scheme of AGN (Antonucci 1993), there is little Doppler boosting of the jets and hotspots due to the orientation of the source with respect to the line of sight. This orientation also allows for studies of the hypothesized dust and gas torus that surrounds the central engine and lies perpendicular to the jet axis. The actual angle that the line of sight makes with the torus determines what features can be seen. Evidence for this torus comes from detections of broad HI absorption lines (Tavlor et al. 1999; Peck et al. 2000; Pihlström. Conwav, \& Vermeulen 2003; Gupta et al. 2006) and free-free absorption (Peck, Tavlor, \& Conway 1999) towards CSOs. $\mathrm{H}_{2} \mathrm{O}$ megamasers also provide clues to circumnuclear tori in nearby AGN (Lo 2005, and references therein).

This torus, and magnetic fields in the shocked, photoionized ISM around the lobes, may also give rise to the large Faraday screens that depolarize emission from most CSOs (Bicknell, Dopita, \& O'Dea 1997). The first detection of significant polarization in two CSOs in the COINS sample (CSOs Observed In the Northern Sky; Peck \& Taylor 2000), $\mathrm{J} 0000+4054(2.1 \%)$ and $\mathrm{J} 1826+1831(8.8 \%)$, occured on the side with the more prominent jet or hotspot (Gugliucci et al. 2005, hereafter GTPG). The opposite hotspot showed no polarization down to a $0.3 \mathrm{mJy}$ limit. Any polarization that is detected should have high rotation measures (several 1000s rad m-2; Bicknell, Dopita, \& O’Dea 1997, and references therein). In this paper, we attempt to calculate the Faraday rotation measures in these polarized components.

Kinematic ages can be obtained by measuring the separation speed between hotspots over time, or the proper motion of a hotspot or jet component with respect to the core (e.g. Polatidis \& Conway 2003; Nagai et al. 2006). In GTPG, we confirmed ages for three CSOs between $130 \pm 47$ and $3000 \pm 1490 \mathrm{yr}$, with another source appearing to have an age of $20 \pm 4$ 
yr. The overall CSO age distribution seems to be disproportionately stacked towards the younger ages. Possible explanations are: there is a selection effect against older CSOs, the jet activity tends to die off after a certain period of time, or CSOs have periods of deactivation and reactivation (Tingay, Edwards, \& Tzioumis 2003). Those that survive may evolve into Fanaroff-Riley II galaxies such as Cygnus A (Fanaroff \& Riley 1974; Readhead et al. 1996b; Polatidis \& Conway 2003). In this paper, we refine the kinematic age estimates of J0000+4054 and J1826+1831 that were first calculated in GTPG. Also included are observations of a new CSO candidate from the Second Caltech-Jodrell Bank Survey, J1915+6548, which also shows hotspot separation over three epochs and polarization in its more prominent hotspot.

Throughout this discussion, we assume $\mathrm{H}_{0}=71 \mathrm{~km} \mathrm{~s}^{-1} \mathrm{Mpc}^{-1}, \Omega_{M}=0.27$, and $\Omega_{\Lambda}=$ 0.73. Linear sizes and velocities for sources with known redshifts have been calculated using E.L. Wright's cosmology calculator 1 .

\section{Observations and Analysis}

Observations were centered on $4.8 \mathrm{GHz}$ and $8.4 \mathrm{GHz}$ on 12 February 2005 for J0000+4054 and on 18 February 2005 for J1826+1831 using the VLBA 2 . Observations of J1915+6548 were centered on $4.8 \mathrm{GHz}, 8.4 \mathrm{GHz}, 15.1 \mathrm{GHz}$, and $22.2 \mathrm{GHz}$ with the VLBA on 11 November 2004. Each frequency was separated into four IFs, and these IFs were paired such that the higher two frequencies were averaged during imaging as were the lower two frequencies, except at $22.2 \mathrm{GHz}$ where all four IFs were averaged. Therefore, the frequencies used for the RMs in these observations were $4.6 \mathrm{GHz}, 5.0 \mathrm{GHz}, 8.2 \mathrm{GHz}, 8.5 \mathrm{GHz}, 14.9 \mathrm{GHz}, 15.3 \mathrm{GHz}$, and $22.2 \mathrm{GHz}$. Observational parameters are presented in Table 1.

Amplitude calibration of the data was derived from system temperatures and antenna gains. Fringe-fitting was performed with the AIPS task FRING on the calibrators OQ 208 and 3C 84. D-term solutions were determined with the AIPS task LPCAL and the same calibrators OQ 208 and 3C 84. Absolute electric vector position angle (EVPA) calibration was determined for J0000+4054 using the EVPAs of J1310+322 and for J1826+1831 and J1915+6548 using the EVPAs of BL Lac listed in the VLA Monitoring Program 3

\footnotetext{
${ }^{1}$ http://www.astro.ucla.edu/ wright/CosmoCalc.html

${ }^{2}$ The National Radio Astronomy Observatory is operated by Associated Universities, Inc., under cooperative agreement with the National Science Foundation.

${ }^{3}$ http://www.vla.nrao.edu/astro/calib/polar/
} 
(Taylor \& Myers 2000). Note that the EVPAs were corrected for each of the four IFs separately.

\section{Results}

Faraday rotation was first noted by Michael Faraday when he passed polarized light through a refractive medium in the presence of a magnetic field (Faraday 1933). The intrinsic polarization angle, $\chi_{0}$, is observed as $\chi$ such that

$$
\chi=\chi_{0}+R M \lambda^{2}
$$

where $\lambda$ is the observed wavelength. The rotation measure, RM, is related to the electron density, $n_{\mathrm{e}}$, the net line of sight magnetic field in the environment, $B_{\|}$, and the path length, $d l$, through the plasma, by the equation

$$
R M=812 \int n_{\mathrm{e}} B_{\|} d l \quad \mathrm{rad} \mathrm{m}^{-2}
$$

where units are in $\mathrm{cm}^{-3}, \mathrm{mG}$, and parsecs. A reasonable $n_{\mathrm{e}}$ for radio galaxies is $10^{3} \mathrm{~cm}^{-3}$ as estimated by Zavala \& Taylor (2003). Our lower limit for the path length is 0.3 pc, the approximate size of clumps within the Faraday screen in M87 (Zavala \& Taylor 2002). Since the Faraday screen for an AGN is now considered to come from interactions of the jet with ambient material (Zavala \& Taylor 2004), a good upper limit for the path length is the jet radius, or 10 pc. Field strengths calculated with these parameters can be compared to the strength of a magnetic field in pressure balance with a thermal gas of the same $n_{\mathrm{e}}$ and a temperature of $10^{4} \mathrm{~K}$ using

$$
\frac{B^{2}}{8 \pi}=n_{\mathrm{e}} k T
$$

The $8.4 \mathrm{GHz}$ images of J0000+4054 and J1826+1831 at full resolution are shown in Figures 1 and 2 with sticks representing polarization vectors. The $8.4 \mathrm{GHz}$ images were then tapered to match the resolution of the $4.8 \mathrm{GHz}$ images. The same circular beam was then applied to both images and spectral index maps were created. These are overlayed with the $4.8 \mathrm{GHz}$ images in Figures 1 and 2. A plot for the rotation measure of the polarized component of J1826+1831 is shown in Figure 3. Source parameters for these CSOs and $\mathrm{J} 1915+6548$ are in Table 1.

In GTPG, we attempted to obtain relative proper motions for CSOs in the COINS sample in order to calculate a kinematic age for each source. This assumes that the separation speed of the hotspots is uniform. This method is independent of the angle that the source 
makes with respect to the line of sight and of the distance to the source. This method also produces results if the hotspot or outer jet component can be seen moving away from the core. Using $8.4 \mathrm{GHz}$ data from three epochs spread across a five year period, we calculated the kinematic ages of three CSOs with reasonable certainty, while the rest provided lower limits. These new observations provided us with a fourth epoch for J0000+4054 and J1826+1831 at $8.4 \mathrm{GHz}$, extending the time baseline to seven years. This provides better estimates and limits of the ages of these two CSOs.

Models for J0000+4054 and J1826+1831 were fit to the visibility data for the March 2000 epoch, since this was close to the middle of the time baseline and had a high dynamic range. These models were comprised of elliptical Gaussians. Visibility data from each epoch were fit to a model with the same size and shape ellipses so that only the positions and fluxes of these were allowed to vary with time. Uncertainties in position for each component were calculated from the signal-to-noise ratios and the synthesized beam. The positions of these components with respect to a reference component were fit with a weighted least squares line where the slope of the line provides the relative speed. Motions are considered significant if they are at least $3 \sigma$ above the errors and if the reduced chi-squared is nearly 1 . Errors are dependent on the image noise and the individual flux of each component. If large errors are present, then the motions are considered upper limits, so that they give rise to ages that are lower limits. Table 3 includes the modelfit parameters, and Figure 4 gives plots of the proper motion of two components in $\mathrm{J} 1826+1831$.

Total intensity images of J1915+6548, the new CSO candidate, are presented in Figure 5 with sticks representing polarization vectors overlayed. The integrated total power spectrum, as well as that of component A alone, is shown in Figure 6. Since the brightest hotspot was polarized at $8.4 \mathrm{GHz}, 15.1 \mathrm{GHz}$, and $22.2 \mathrm{GHz}$, a rotation measure plot is given in Figure 7. Figure 8 presents the proper motions of hotspot separation in this source at $4.8 \mathrm{GHz}$ over an 11 year timespan. Table 4 gives hotspot brightnesses for the polarized sources and the unpolarized sources of the COINS sample for comparison in $\S 4$. Plots of $\beta$ vs. $\theta$ for the separation speeds and orientations of J1826+1831 and J1915+6548 are presented in Figure 9.

\section{1. $\mathrm{J} 0000+4054$}

This CSO was identified as such in Dallacasa et al. (2002) and GTPG, and polarization was detected at $8.4 \mathrm{GHz}$. It is associated with a galaxy of magnitude 21.4 (Stickel \& Kuehr 1996). The core has still not been positively identified from the spectral index map (see Fig. 1). From their compact morphologies, either B1 or B2 could be the core, but their spectral indices are rather steep $\left(\alpha \approx-0.6 ; S_{\nu} \propto \nu^{\alpha}\right)$. However, there appears to be a flattening 
$(\alpha \approx-0.4)$ between components B1 and B2, so the core may be located there (see inset Fig. 1.b). In Dallacasa et al. (2002), the combined flux of B1 and B2 (component Ce in that paper) is $71 \mathrm{mJy}$ at $1.6 \mathrm{GHz}$. When compared with the fluxes of B1 and B2 here at $8.4 \mathrm{GHz}$, this yields a spectral index for that region of $\alpha \approx-0.25$. High dynamic range imaging at $15 \mathrm{GHz}$ should provide a positive identification.

Polarization was detected in the southern hotspot at $8.4 \mathrm{GHz}$ with an intensity of $2.1 \mathrm{mJy}$ (see Fig. 1). This is $1.2 \%$ of the intensity of the hotspot. There is no detectable polarization at $4.8 \mathrm{GHz}$ down to the $3 \sigma$ level $(\sim 0.2 \mathrm{mJy}$, or $0.06 \%)$. The $4.8 \mathrm{GHz}$ image also shows a strange morphology in the southern lobe. There appears to be a hole, or depression, in the emission below the bright hotspot. If this is not an artifact in the data, it resembles the region in the eastern lobe of 4C 31.04 by Giroletti et al. (2003), where it is speculated that such a hole could be created by a dense molecular gas that is impenetrable to radio emission or to the plasma itself. However, the morphology may also be the effect of edge-brightening at component $\mathrm{C}$ if the plasma is running into denser material. This material may also be deflecting the jet to the east, as seen in the $8.4 \mathrm{GHz}$ image. Polarization was only detected at $8.4 \mathrm{GHz}$, so the RM could not be determined and the true orientation of the magnetic field is not known. If the RM was known to be as low as in $\mathrm{J} 1826+1831\left(-180 \mathrm{rad} \mathrm{m}^{-2}\right.$, see $\S 3.2$ ), for example, then there would be little rotation from the original polarization angle to $8.4 \mathrm{GHz}$, so the magnetic field would lie roughly perpendicular to the electric vectors in Figure 1a. Then, the detectable polarization in this region would be due to a compression of the ambient magnetic field at a shock front where the jet is colliding with the denser gas. Future observations at $15 \mathrm{GHz}$ should allow us to determine the rotation measure for this region.

The fourth epoch of $8.4 \mathrm{GHz}$ data in the proper motion study of this object did not provide a good fit for the separation speed of the hotspots. However, there is an upper limit of 0.066 mas $\mathrm{yr}^{-1}$ of component $\mathrm{A}$ away from component $\mathrm{C}$. This speed limit and a hotspot distance of 40.33 mas provides a lower limit for the kinematic age of $610 \mathrm{yrs}$. This is higher than was previously estimated (280 yrs in GTPG). Although the redshift is not known, a reasonable estimate of $z \approx 0.5$ can be made for the typical redshift of a CSO host (Augusto et al. 2006). If this is the case, the projected distance between components A and $\mathrm{C}$ is $242 \mathrm{pc}$ and $\mathrm{A}$ is moving with speed $v<2.0 \mathrm{c}$.

\subsection{J1826+1831}

This is the most significantly polarized of these three objects. Component C, most likely a jet component, has a polarized intensity of $2.3 \mathrm{mJy}$ at $8.4 \mathrm{GHz}$, or $8.5 \%$ of its flux (see 
Fig. 2). This component has a polarized intensity of $1.4 \mathrm{mJy}$ at $4.8 \mathrm{GHz}$, making it $3.7 \%$ polarized. A least-squares fit for the rotation measure of $-180 \pm 10 \mathrm{rad} \mathrm{m}^{-2}$ at the peak of component $\mathrm{C}$ is presented in Figure 3. This is not an uncommon rotation measure for a typical quasar jet (Zavala \& Taylor 2004). The pair of angles at 8.2 and $8.5 \mathrm{GHz}$ suggest a higher rotation measure, but this requires for a number of 180 degree turns to be put in between 4.6 and $5.0 \mathrm{GHz}$ and between $5.0 \mathrm{GHz}$ and $8.2 \mathrm{GHz}$. These turns can be introduced because the polarization vectors give an orientation, but not a direction, of the electric field such that $\chi$ and $\chi \pm 180$ are indistinguishable. Depending on the number of turns used in this data, rotation measures as high as $6000 \mathrm{rad} \mathrm{m}^{-2}$ are plausible. One must take caution with this, however, since any number of turns can be introduced to provide a false good fit. Therefore, $-180 \pm 10 \mathrm{rad} \mathrm{m}^{-2}$ is a conservative estimate. Measurement of polarization at another frequency may help in determining the correct rotation measure. It may also be true, however, that we only see polarized emission from this component because it is free of the high rotation measures that may be present closer to the core.

If a path length of $0.3 \mathrm{pc}$ is assumed with $\mathrm{n}_{e}=10^{3} \mathrm{~cm}^{-3}$, the magnetic field strength is $0.7 \mu \mathrm{G}$, using Eqn. 2. With the upper limit of the path length, $10 \mathrm{pc}$, the field strength is only $0.03 \mu \mathrm{G}$. Both of these are much smaller than the strength of a magnetic field that is in pressure balance with a $10^{4} \mathrm{~K}$ gas of the same electron density, $\sim 200 \mu \mathrm{G}$, using Eqn. 3 .

A spectral index map was made between 4.8 and $8.4 \mathrm{GHz}$ which is presented in Figure 2. This shows the core, $\mathrm{B}$, to be a flat spectrum component $(\alpha \approx 0.6)$, while the rest of the jet and counterjet are steep spectrum $(\alpha \approx-0.6$ to -1.7$)$.

The proper motions of components $\mathrm{C}$ and $\mathrm{D}$ with respect to $\mathrm{B}$ were measured using the

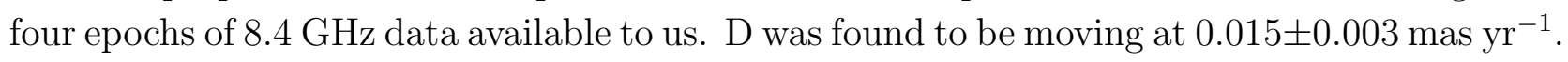
With a projected separation between $\mathrm{B}$ and $\mathrm{D}$ of 41.87 mas, this corresponds to a kinematic age for the CSO of $2600 \pm 490 \mathrm{yr}$. This refines our earlier estimate of $3000 \pm 1490 \mathrm{yr}$. The jet

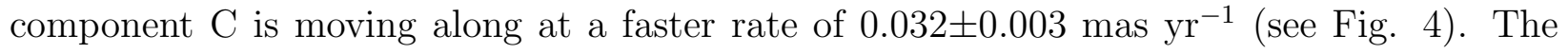
modelfitting of components works best for bright, compact components, of which component $\mathrm{A}$ is neither, so no proper motions can be fit. If we assume again that $z \approx 0.5, \mathrm{D}$ is moving away from $\mathrm{B}$ at $\sim 0.45 c$ and is $255 \mathrm{pc}$ away. $\mathrm{C}$ is moving away from $\mathrm{B}$ at $\sim 0.95 c$. However, this source has no detected optical counterpart, so its redshift could be much higher.

\subsection{J1915+6548}

This new CSO candidate was first listed in a survey with the 300-ft Green Bank Telescope at $4.8 \mathrm{GHz}$ by Becker. White, \& Edwards (1991) It was observed in the Sec- 
ond Caltech-Jodrell Bank Survey (CJ2) which presented a spectrum of total intensity in Henstock et al. (1995). Its host is a Seyfert 1 galaxy with a magnitude of 18.2 and redshift of $z=0.486$ (Henstock et al. 1997). New observations were taken with the VLBA to investigate the non-linear morphology in the CJ2 image.

We identify Component $\mathrm{A}$ as a steep spectrum hotspot $(\alpha \approx-0.9$; See Fig. 6$)$ that dominates the flux of this object at all four frequencies. Components $\mathrm{B}$ and $\mathrm{C}$ are steep spectrum $(\alpha \approx-1.2$ and -0.9 , respectively), and we propose that they are also part of the eastern lobe. Component $\mathrm{D}$ is very steep spectrum $(\alpha \approx-1.6)$, and, based on morphology, we propose it to be the counter-hotspot to A. There is no evidence of a compact, flat or inverted spectrum core, but there are a growing number of CSOs that have two steep spectrum hotspots but no visible core (e.g. J0620+2102, J1111+1955; GTPG). There is also a trail of extended emission between $\mathrm{A}$ and $\mathrm{D}$ at 4.8 and $8.4 \mathrm{GHz}$ that hints at a jet-like structure, as well as emission further east of $\mathrm{A}$ in the $4.8 \mathrm{GHz}$ image which we interpret as a sign of earlier activity (see Fig. 5). Although the morphology is similar to that of a CSO, the hotspot intensity ratio at $4.8 \mathrm{GHz}$ is $12: 1$ and is $26: 1$ at $8.4 \mathrm{GHz}$. These fall short of the 10:1 criteria set for CSOs in the COINS sample (Peck \& Taylor 2000).

To determine the synchrotron age of the source, we present the total intensity spectrum of J1915+6548 in Figure 6 using total intensities from this paper and data points from the NASA/IPAC Extragalactic Database (NED) 4 . Using the break frequency, $17 \mathrm{GHz}$, the minimum energy magnetic field of the plasma can be calculated as in Miley (1980) using

$$
B_{m e}=1.4 \times 10^{-4}(1+z)^{1.1} \nu_{0}^{0.22}\left(\frac{F_{0}}{\theta_{x} \theta_{y} s}\right)^{2 / 7}
$$

where $\mathrm{B}$ is in gauss, $\nu_{0}$ is in $\mathrm{GHz}, \mathrm{F}_{0}$ is in $\mathrm{Jy}, \theta_{x}$ and $\theta_{y}$ are the dimensions of an elliptical component in arcseconds, and $s$ is the path length in kpc. With this, the synchrotron age of the source can be calculated as in Murgia et al. (1999) with

$$
\tau_{\text {syn }}=\frac{1610 B^{0.5}}{\left[B^{2}+B_{C M B}^{2}\right]\left[\nu_{b r}(1+z)\right]^{1 / 2}}
$$

with $\mathrm{B}$ in $\mu \mathrm{G}, \nu_{b r}$ in $\mathrm{GHz}$, and $B_{C M B}=3.25(1+z)^{2}$. At $4.8 \mathrm{GHz}$, the eastern hotspot is $0.291 \mathrm{Jy}$ and can be approximated by an ellipse 0.01 mas by 0.006 mas. We assume the path length to be equivalent to $0.01 \mathrm{mas}$, or $0.07 \mathrm{kpc}$ at a redshift of 0.486 . This yields a magnetic field of $\sim 7 \mathrm{mG}$ and a synchrotron age of $\sim 540$ yrs.

Component $\mathrm{A}$ is polarized at $8.4 \mathrm{GHz}(3.0 \mathrm{mJy}$ or $1.6 \%), 15.1 \mathrm{GHz}(2.3 \mathrm{mJy}$ or $2.8 \%)$, and $22.2 \mathrm{GHz}(1.8 \mathrm{mJy}$ or $4.0 \%)$. The polarized flux is too low to be reliable at $4.8 \mathrm{GHz}$

\footnotetext{
${ }^{4}$ NED is operated by the Jet Propulsion Laboratory, Caltech, under contract with NASA.
} 
(1.3 mJy or $0.5 \%)$. A plot of the rotation measure is given in Figure 7 . The rotation measure of $1540 \pm 7 \mathrm{rad} \mathrm{m}^{-2}$ comes from a direct measurement of the EVPAs at the center of the polarized component without any introduced 180 degree wraps. This is more consistent with the high RMs expected from a CSO. With a path length of $0.3 \mathrm{pc}$ and the same electron density as above, this corresponds to a magnetic field strength of $6 \mu \mathrm{G}$. At the upper limit for path length of $10 \mathrm{pc}$, the magnetic field strength is $0.2 \mu \mathrm{G}$. These are still much lower than what is expected in the central regions of a radio galaxy $(200 \mu \mathrm{G}$, see $\S 3.2)$.

The visibility data at $4.8 \mathrm{GHz}$ was modelfit at this epoch and at two other epochs, 1993.444 and 1995.691, the first of which was presented in Henstock et al. (1995). Proper motions were fit with a least squares line with a slope of $0.035 \pm 0.004$ mas $\mathrm{yr}^{-1}$. With a redshift of $z=0.486$, this apparent speed corresponds to $1.02 \pm 0.11 \mathrm{c}$. Although this is higher than typical hotspot separation speeds (GTPG), it yields a good fit (see Fig. 8) and a kinematic age of $940 \pm 110 \mathrm{yr}$.

\section{Discussion}

\subsection{CSO Orientations}

Depolarization in CSOs is consistent with the Bicknell, Dopita, \& O'Dea (1997) model. Variations in rotation measure across the lobe can cause this depolarization, and this may be due to magnetic field reversals in the post-shock ISM. The obscuring torus may also play a role in depolarization, and this would be dependent on torus scale height, opening angle, and orientation. We rule out depolarization by a thin disk of material, with a scale height much less than the radio source size, since we frequently see that both sides are depolarized, whereas a thin disk would only cover one side. If a Faraday screen is to produce the observed polarization asymmetry in these sources, then it requires a scale height of about half the total source size. More detections of polarization in CSOs are needed to constrain its geometry.

The existence of polarized emission in these three sources sets them apart from the rest of the COINS sample. However, this is not the only difference. In GTPG, we noted that J0000+4054 and J1826+1831 appeared to have greater hotspot intensity ratios than their unpolarized counterparts. Since the polarization is detected on the side of the more prominent hotspot, this can be attributed to a shorter path length through the circumnuclear torus and, consequently, a lower Faraday depth. Table 4 lists the brightnesses of each hotspot and the ratio of the dimmer one to the brighter one for unpolarized and polarized CSOs. There is a bimodal distribution in that the polarized sources presented in this paper have ratios $\leq 0.1$ and the unpolarized COINS have ratios $\geq 0.3$. Although the sample size for 
polarized CSOs is small, this is consistent with the observed polarization being due to an orientation effect. As the angle between the jet axis and line of sight decreases, the forward hotspot may be more Doppler boosted and the receding hotspot Doppler dimmed, assuming mildly relativistic bulk motions. So, the leading hotspots in the three polarized sources are oriented such that they are relatively free from Faraday depolarization by the torus that surrounds the central region. However, to explain the depolarization over many viewing angles in the other CSOs, a large torus is needed to extend over hundreds of parsecs of jets and lobe. HI absorption has been seen at this distance from the central engine (e.g. 1946+708 Peck, Taylor, \& Conway 1999).

In order to quantify depolarization in CSOs, we look at Faraday beam depolarization. We can calculate the necessary rotation measure gradient across the synthesized beam in order to get a rotation of one radian. For the unpolarized CSOs in the COINS sample, with an average beamwidth of 1.5 mas at $8.4 \mathrm{GHz}$, the RM gradient need only be $\sim 500 \mathrm{rad} \mathrm{m}^{-2} \mathrm{mas}^{-1}$. So, the RM of J1915+6548 may be reasonable for a CSO, whereas the low rotation measure of $\mathrm{J} 1826+1831$ is still puzzling. An RM of $500 \mathrm{rad} \mathrm{m}^{-2}$ corresponds to a magnetic field strength of $2 \mu \mathrm{G}$ with a path length of $0.3 \mathrm{pc}$ and $0.06 \mu \mathrm{G}$ with $10 \mathrm{pc}$. These are still much lower than the strength of a magnetic field in pressure balance with a $10^{4} \mathrm{~K}$ gas.

We use the assumption that relativistic beaming is in effect to quantify the orientations of these sources. We can match up what appear to be jet components from the approaching and receding sides of the core and compare their fluxes $\left(S_{a}\right.$ and $\left.S_{r}\right)$ as

$$
\frac{S_{a}}{S_{r}}=\left(\frac{1+\beta \cos \theta}{1-\beta \cos \theta}\right)^{n-\alpha}
$$

where $\beta$ is the space velocity, $\theta$ is the angle to the line of sight, $\alpha$ is the spectral index, and $n$ is either 2 or 3 . Models for continuous jets are best fit by $n=2$ and jets of discrete components by $n=3$. One can use

$$
\frac{\mu_{a}}{\mu_{r}}=\frac{d_{a}}{d_{r}}=\frac{1+\beta \cos \theta}{1-\beta \cos \theta}
$$

where $\mu$ is the apparent motion and $d$ is the distance from the core. However, this requires a well known position for the center of activity. One can also use the hotspot separation, $\mu_{\text {sep }}=\left|\mu_{a}\right|+\left|\mu_{r}\right|$, such that

$$
v_{\text {sep }}=\mu_{\text {sep }} D_{a}(1+z)=\frac{2 \beta \sin \theta}{1-\beta^{2} \cos ^{2} \theta},
$$

where $D_{a}$ is the angular size distance to the source, $z$ is the redshift, and $v_{\text {sep }}$ is the angular separation speed in units of $c$ (Taylor \& Vermeulen 1997). These can provide constraints on $\beta$ and $\theta$. 
We know neither the core location nor the redshift for J0000+4054. So, only Eqn. 6 can be applied, using the fluxes of the hotspots as determined by the model in Table 3 . A locus of $\beta$ and $\theta$ is plotted in Figure 9a. Note that a lower limit is plotted when $n=2$ and an upper limit when $n=3$. The errors in the measured fluxes are negligible compared to this spread. This shows that, approximately, $\beta \geq 0.3$ for all values of $\theta$.

For J1826+1831, we used the brightnesses of each hotspot, components A and D, with Eqn. 6 as well as their distances from the core with Eqn. 7 to calculate loci of $\beta$ and $\theta$ (Figure 9b). Note that these two equations have a similar functional form, so no tight constraints can be determined. This gives an upper limit for the angle to the line of sight of $\sim 75^{\circ}$. The true space velocity will be $\geq 0.3 c$ for any angle. This is higher than typical values found for other CSOs $(\sim 0.1 c$ Owsianik \& Conway 1998) and other radio galaxies $\left(<0.1 c\right.$ Scheuer 1995). If the value for $\mu_{\text {sep }}$ of component D $\left(0.015 \mathrm{mas} \mathrm{yr}^{-1}\right)$ is used in Eqn. 8 with a typical CSO redshift $(z \approx 0.5)$, the projected separation velocity is $\sim 0.4 c$. This is consistent with our results. J1826+1831 cannot be very close (for example, $z=0.01$ ), for then the separation velocity would be unusually low and inconsistent with Figure $9 \mathrm{~b}$.

We used the apparent hotspot separation speed of $1.02 \pm 0.11 c$ for J1915+6548, $z=0.486$, and Eqn. 8 to plot the locus for $\beta$ and $\theta$ in Figure 9c along with the loci for the two brightness models with Eqn. 6. These two sets of curves intersect when $\beta \approx 0.55$ and $\theta \approx 50^{\circ}$. Such a high space velocity is uncharacteristic of lobes. However, it is possible that the hotspot being measured is a transient feature, and therefore moving more quickly, whereas the lobe as a whole is moving at a much slower speed. This can occur if the jet that is feeding the hotspot has changed its orientation slightly and is drilling out a new part of the lobe (Scheuer 1995). Alternatively, we may be measuring the separation of jet components rather than hotspots as there may be no visible hotspots. This example shows how the orientation angle of a CSO can be measured if mild relativistic beaming is a factor.

\subsection{CSO Environments}

An alternate theory for the small sizes of CSOs is that they are old sources frustrated by a dense medium (Carvalho 1994). This dense medium may in fact be asymmetric, possibly as a result of a galaxy merger or interaction (Carvalho 1998). In an extreme case, the ages of these sources would be comparable to the ages of larger, classic radio doubles. Readhead et al. (1996a) pointed out that if this were the case, CSOs would have more spherical morphologies. Also, hotspot separation speeds in Polatidis \& Conway (2003) and GTPG show that the hotspots are still moving too quickly to be confined by a dense medium and be older than a few thousand years. However, this does not rule out high densities or 
asymmetries in the medium around CSOs as evidence by Orienti, Dallacasa, \& Stanghellini (2006). Bicknell, Dopita, \& O'Dea (1997) propose that CSOs are frustrated but not confined by the interstellar medium (ISM). Their model assumes a dentist-drill explanation for jet-lobe interactions and predicts low polarization. This is because the ionized gas surrounding the lobes in this model produces large variations in Faraday rotation measures across the source, thus depolarizing the emission. Interstellar magnetic fields can play an integral role if there are a large number of magnetic field reversals across the source which would produce a varied RM structure in our maps. Since the polarization is spatially isolated in these CSOs, no such maps can be made.

Asymmetries in the CSO environment may cause one hotspot to be considerably more polarized in these few sources by interactions with a dense ISM. If the EVPAs for J1915+6548 are extrapolated back to zero wavelength, the observed angle of the electric field is $-88^{\circ}$. So, the orientation of the magnetic field in the image would be nearly north-south, which is approximately perpendicular to the source orientation. This may be an indication of a collision between the bright hotspot and a dense medium that orders the magnetic field along the axis of compression. This is also observed in J1826+1831 where the EVPA at zero wavelength is $75^{\circ}$, so the magnetic field is oriented at $-25^{\circ}$. This is also nearly perpendicular to the source orientation, but this effect is for the bright jet component, not the hotspot. Components may also be brighter (by Doppler boosting) if they are moving more quickly through a less dense ISM. However, the indistinguishable motions of the polarized component in $\mathrm{J} 0000+4054$ seems to refute that.

This asymmetry in environments does not, however, explain the bimodal distribution in Table 4, that is, the fact that the ratios of the dimmer hotspots to the brighter hotspots of each source are much lower for polarized CSOs than for unpolarized CSOs. This is more naturally explained by Doppler boosting. Also, the relativistic hotspot speeds of J1915+6548 (see Fig. 9) could not exist in a dense environment. Finally, we do not see rotation measures in the several thousands as predicted by the Bicknell. Dopita, \& O'Dea (1997) model.

\section{Conclusions}

In this study we have investigated the polarization properties of two CSOs, J0000+4054 and J1826+1831, and a CSO candidate J1915+6548. The Faraday rotation measures that were observed for J1826+1831 and J1915+6548 are lower than what was previously expected for CSOs, $-180 \pm 10 \mathrm{rad} \mathrm{m}^{-2}$ and $1540 \pm 7 \mathrm{rad} \mathrm{m}^{-2}$, compared to predicted values of several thousand $\mathrm{rad} \mathrm{m}^{-2}$. These imply low external magnetic field strengths, 0.03 to $6 \mu \mathrm{G}$, depending on what estimates for electron density and path length are used. However, only 
about $500 \mathrm{rad} \mathrm{m}^{-2} \mathrm{mas}^{-1}$ is needed for beam depolarization to be a major factor for most unpolarized CSOs.

Since these polarized sources have significantly more asymmetric hotspot brightnesses than their unpolarized counterparts, with ratios of $\leq 0.1$ as opposed to $\geq 0.3$, it is plausible that Doppler boosting is in effect for the brighter hotspots. Then, their jet axis orientations are closer to the line of sight than for other CSOs such that the polarized hotspot is free of obscuration from the circumnuclear torus. We put contraints on $\beta$ and $\theta$ for all three sources (Figure 9) such that for J0000+4054 and J1826+1831, $\beta \geq 0.3$. More information, specifically the redshift, must be known in order to make tighter constraints. For J1915+6548, $\beta \approx 0.5$ and $\theta \approx 50^{\circ}$.

We extended the time baseline for proper motions of the two objects from the COINS sample from five to seven years. This provided a better age estimate for J1826+1831 of $2600 \pm 490$ yrs and a better lower limit for the age of J0000+4054 at 610 yrs. Extending the time baseline for the proper motions of a larger sample of CSOs will reduce some of the uncertainties and lower limits of their ages so that the distribution of ages in the sample can be better determined.

J1915+6548 was determined to be a CSO candidate because of the presence of symmetric, steep spectrum hotspots and the lack of a compact, flat or inverted spectrum core. If confirmed, this will be the third CSO with significant polarization. Although spectral age arguments and proper motions from an eleven year time baseline suggest an age between approximately 600 and 1000 yrs, the hotspot separation speed is most certainly relativistic and Doppler boosting is occuring in the brighter hotspot. Our age estimates may be underestimating the true source age if the brightest hotspot is undergoing renewed activity from a slowly moving jet or if it is encountering a particularly dense medium, reaccelerating the electrons and giving a lower synchrotron age. If it were the case that the hotspots are transient features and therefore appear younger and faster moving than the radio lobe, then the kinematic ages of many CSOs would be underestimated. However, with such small sources, it is possible that a true classical radio lobe has not yet been created and the hotspots are the only indicator of the current phase of activity in CSOs.

NEG gratefully acknowledges support from the NRAO Graduate Summer Student Research Assistantship. ABP thanks NRAO for travel support and hospitality during part of this project. This research has made use of the NASA Astrophysics Data System. 


\section{REFERENCES}

Antonucci, R. 1993, ARA\&A, 31, 473

Augusto, Pedro, Gonzalez-Serrano, J. Ignacio, Perez-Fournon, Ismael, Wilkinson, Peter N. 2006, MNRAS, 368, 1411

Beasley, A. J., Gordon, D., Peck, A. B., Petrov, L., MacMillan, D. S., Fomalont, E. B., \& Ma, C. 2002, ApJS, 141, 13

Becker, R. H., White, R. L., \& Edwards, A. L. 1991, ApJS, 75, 1

Bicknell, G. V., Dopita, M. A., \& O’Dea, C. P. O. 1997, ApJ, 485, 112

Carvalho, J. C. 1994, A\&A, 292, 392

Carvalho, J. C. 1998, A\&A, 329, 845

Conway, J. E., Myers, S. T., Pearson, T. J., Readhead, A. C. S., Unwin, S. C., \& Xu, W. 1994, ApJ, 425, 568

Dallacasa, D., Tinti, S., Fanti, C., Fanti, R., Gregorini, L., Stanghellini, C., \& Vigotti, M. 2002, A\&A, 389, 115

Fanaroff, B. L. \& Riley, J. M. 1974, MNRAS, 167, 31P

Faraday, M. 1933 in Faraday's Diary, ed. T. Martin (London: G. Bell \& Sons), 264

Giroletti, M., Giovannini, G., Taylor, G. B., Conway, J. E., Lara, L., \& Venturi, T. 2003, A\&A, 399, 889

Gugliucci, N. E., Taylor, G. B., Peck A. B., \& Giroletti M. 2005, ApJ, 622, 136

Gupta, Neeraj, Salter, C. J., Saikia, D. J., Ghosh, T., \& Jeyakumar, S. 2006, MNRAS, 373, 972

Henstock, D. R., Browne, I. W. A., Wilkinson, P. N., Taylor, G. B., Vermeulen, R. C., Pearson, T. J., \& Readhead, A. C. S. 1995, ApJS, 100, 1

Henstock, D. R., Browne, I. W. A., Wilkinson, P. N., McMahon, R. G. 1997, MNRAS, 290, 380

Lo, K.Y. 2005, ARA\&A, 43, 625

Miley, G. 1980, ARA\&A, 18, 165 
Murgia, M., Fanti, C., Fanti, R., Gregorini, L., Klein, U., Mack, K.-H., \& Vigotti, M. 1999, A\&A, 345, 769

Nagai, Hiroshi, Inoue, Makoto, Asada, Keiichi, Kameno, Seiji, \& Doi, Akihiro 2006, ApJ, 648, 148

Orienti, M., Dallacasa, D., \& Stanghellini, C. 2006, A\&A, in press

Owsianik, I. \& Conway, J. E. 1998, A\&A, 337, 690

Peck, A. B., Taylor, G. B., \& Conway, J. E. 1999, ApJ, 521, 103

Peck, A. B. \& Taylor, G. B. 2000, ApJ, 534, 90

Peck, A. B., Taylor, G. B., Fassnacht, C. D., Readhead, A. C. S., Vermeulen, R. C . 2000, ApJ, 534, 104

Pihlström, Y. M., Conway, J. E., \& Vermeulen, R. C. 2003, A\&A, 404, 871

Polatidis, A. G. \& Conway, J. E. 2003, Publications of the Astronomical Society of Australia, 20,69

Readhead, A. C. S., Taylor, G. B., Xu, W., Pearson, T. J., Wilkinson, P. N., \& Polatidis, A. G. 1996a, ApJ, 460, 612

Readhead, A. C. S., Taylor, G. B., Pearson, T. J., \& Wilkinson, P. N. 1996b, ApJ, 460, 634

Scheuer, P. A. G. 1995, MNRAS, 277, 331

Stickel, M. \& Kuehr, H. 1996, A\&AS, 115, 11S

Taylor, G. B., O’Dea, C. P., Peck, A. B. \& Koekemoer, A. M. 1999, ApJ, 512, L27

Taylor, G. B. \& Myers, S. T. 2000 VLBA Scientific Memo 26, National Radio Astronomy Observatory

Taylor, G. B. \& Vermeulen, R. C. 1997, ApJ, 485, 9

Tingay, S. J., Edwards, P. G., \& Tzioumis, A. K. 2003, MNRAS, 346, 327

Wilkinson, P. N., Polatidis, A. G., Readhead, A. C. S., Xu, W., \& Pearson, T. J. 1994, ApJ, 432, L87

Zavala, R. T. \& Taylor, G. B. 2002, ApJL, 566, 9 
Zavala, R. T. \& Taylor, G. B. 2003, ApJ, 589, 126

Zavala, R. T. \& Taylor, G. B. 2004, ApJ, 612, 749 

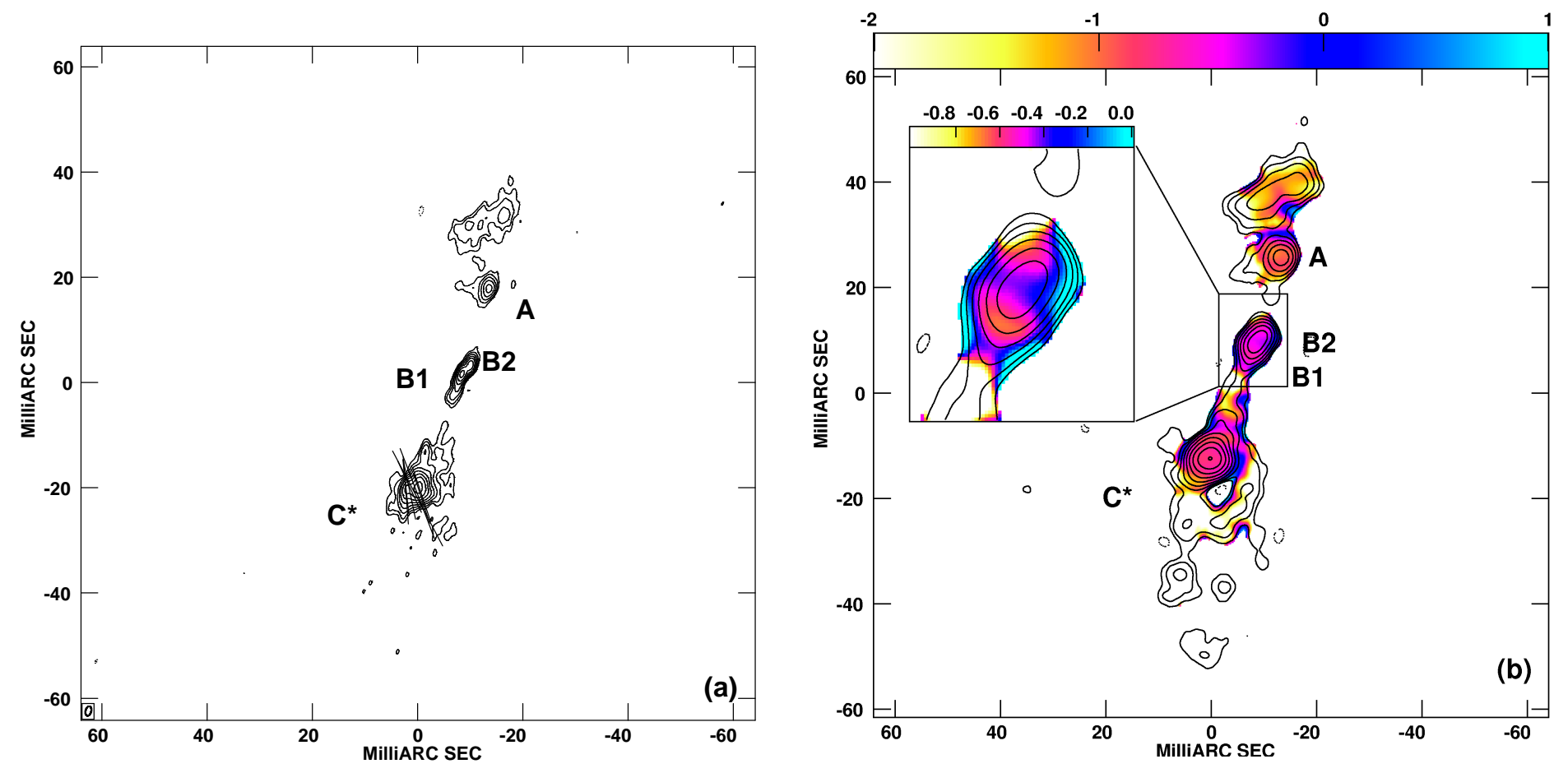

Fig. 1. - Total intensity contours of J0000+4054. (a) Electric polarization vectors over 8.4 GHz contours. A vector length of 1 mas corresponds to a polarized flux density of 0.05 mJy beam ${ }^{-1}$; the smallest ticks correspond to $\sim 0.6 \mathrm{mJy}_{\text {beam }}{ }^{-1}$. Contour levels begin at $0.25 \mathrm{mJy}_{\text {beam }}^{-1}$ and increase by factors of 2. (b) Spectral index map over $4.8 \mathrm{GHz}$ contours where $S_{\nu} \propto \nu^{\alpha}$. Contour levels begin at $0.7 \mathrm{mJy}$ beam $^{-1}$ and increase by factors of 2. The inset rescales the spectral indices for the B1 and B2 components. A star indicates the reference component for motions. 

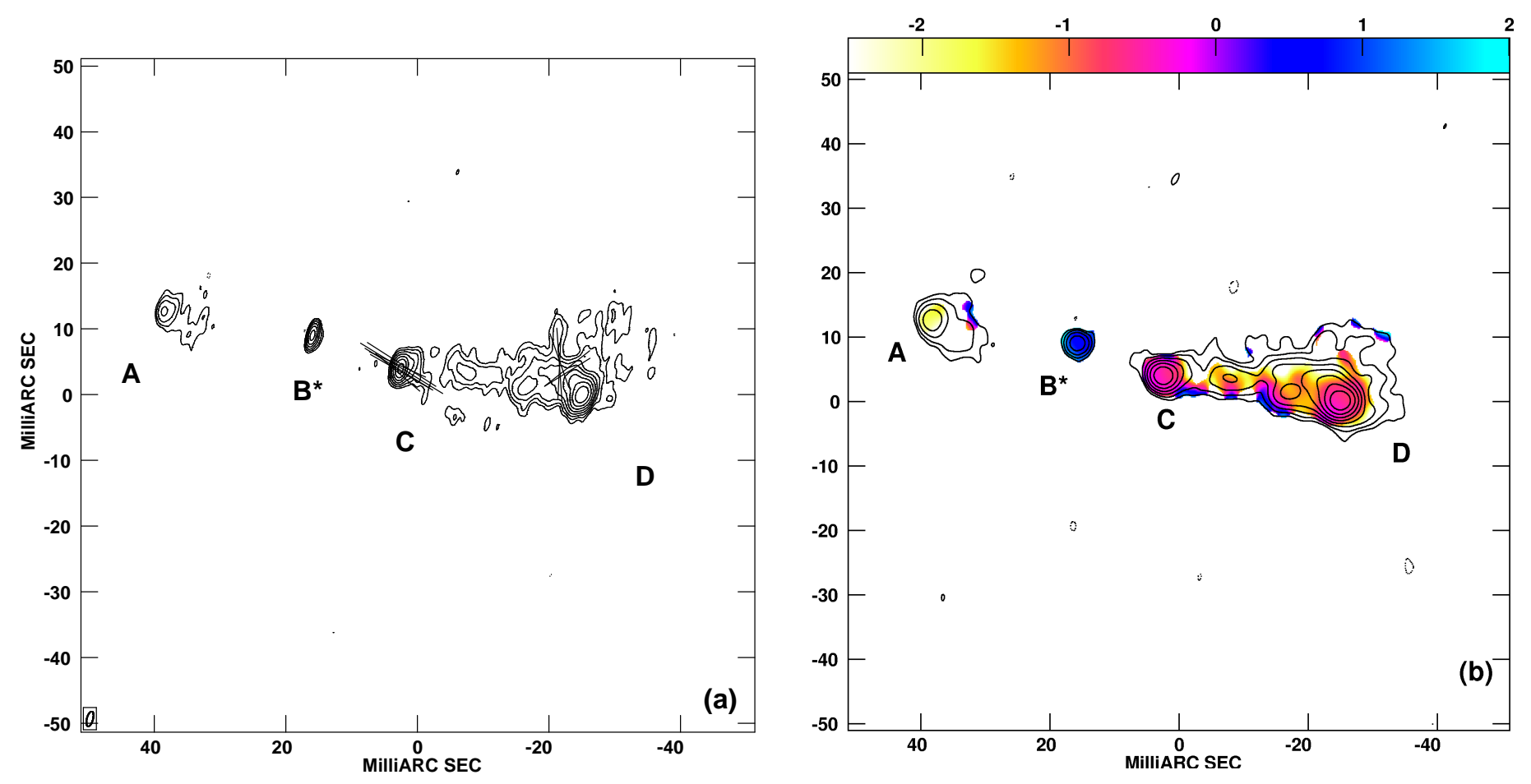

Fig. 2.- Total intensity contours of J1826+1831. (a) Electric polarization vectors over 8.4 GHz contours. A vector length of 1 mas corresponds to a polarized flux density of 0.10 mJy beam $^{-1}$; the smallest ticks correspond to $\sim 0.8 \mathrm{mJy}_{\text {beam }}{ }^{-1}$. Contour levels begin at $0.25 \mathrm{mJy}$ beam $^{-1}$ and increase by factors of 2. (b) Spectral index map over $4.8 \mathrm{GHz}$ contours where $S_{\nu} \propto \nu^{\alpha}$. Contour levels begin at $0.6 \mathrm{mJy}_{\text {beam }}{ }^{-1}$ and increase by factors of 2 . A star indicates the reference component for motions. 


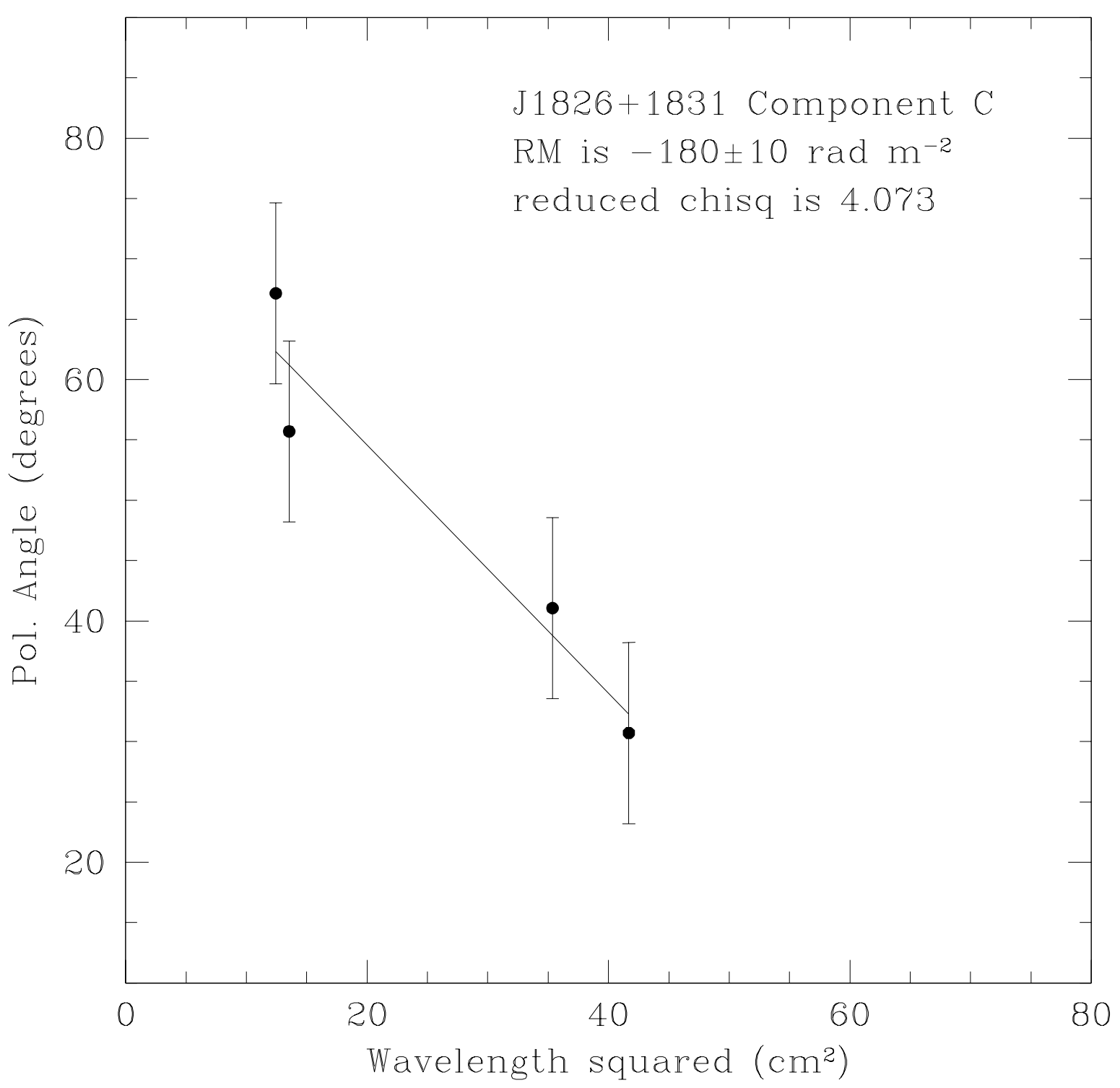

Fig. 3.- Plot of polarization angle versus wavelength squared for component $\mathrm{C}$ of J1826+1831 where the slope of the least squares line is the rotation measure. 

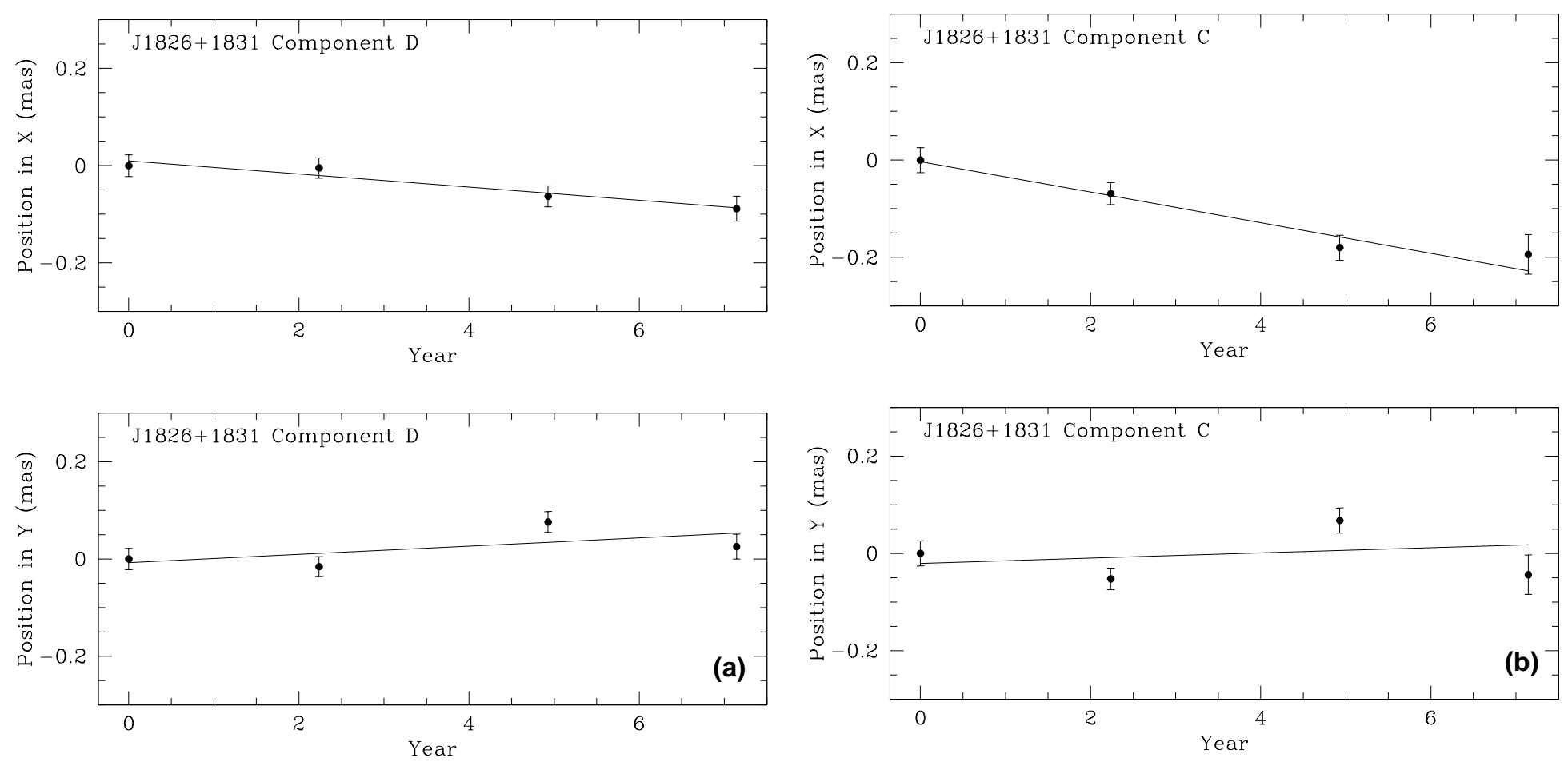

Fig. 4.- Proper motion plots of J1826+1831 with four epochs of $8.4 \mathrm{GHz}$ models to the visibility data. (a) The slope for component $\mathrm{D}$ along the $x$ axis is $-0.014 \pm 0.002{\mathrm{mas} \mathrm{yr}^{-1}}^{-1}$ with a reduced chi squared of 0.912 . The slope along the $y$ axis is $0.008 \pm 0.004{\mathrm{mas} \mathrm{yr}^{-1}}^{-1}$ with a reduced chi squared of 7.487. (b) The slope for component $\mathrm{C}$ along the $x$ axis is

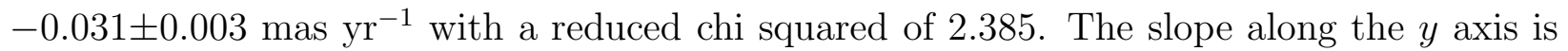

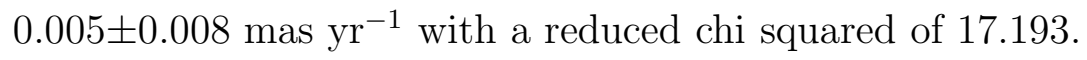



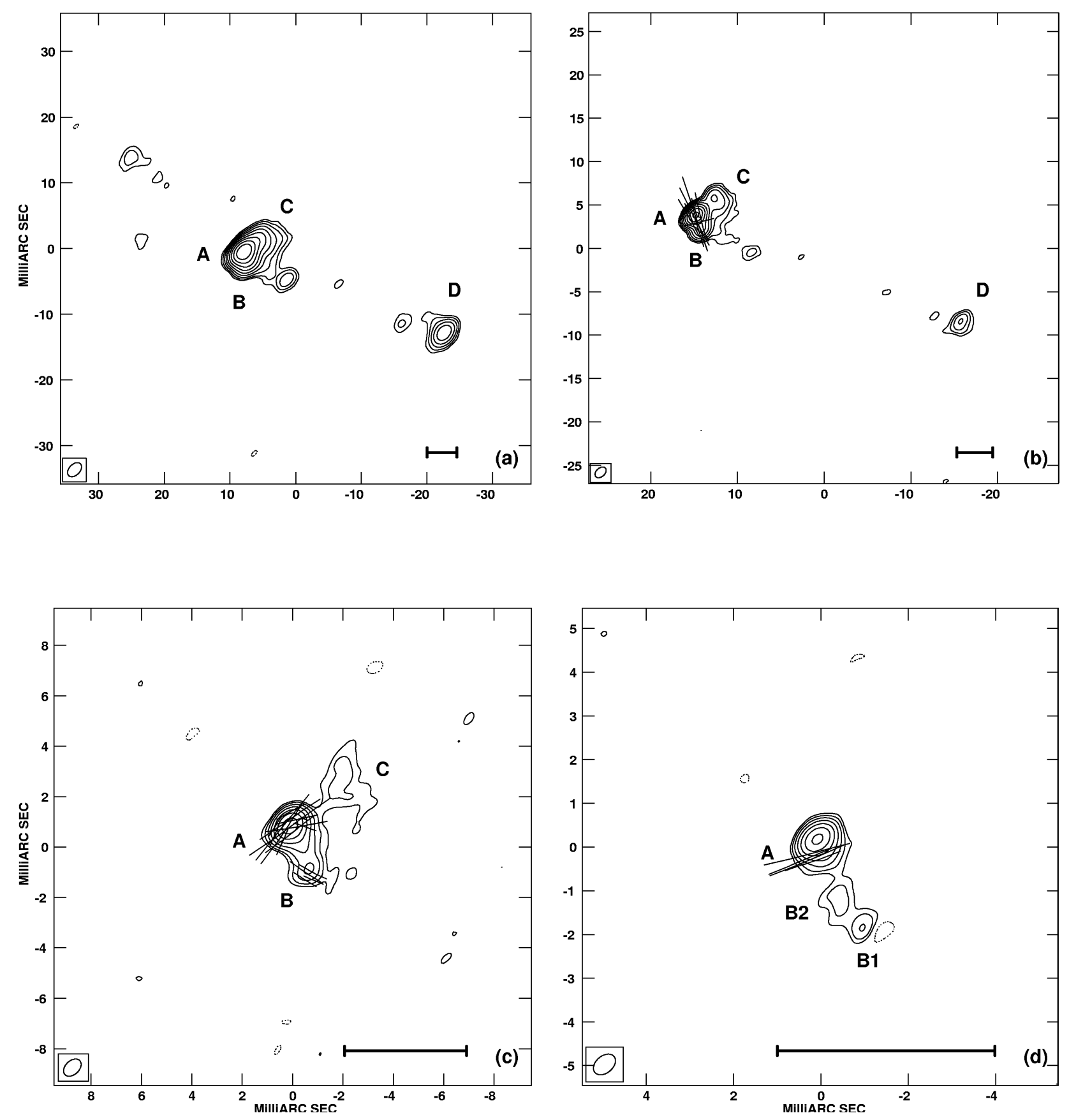

Fig. 5.- Total intensity plots of J1915+6548 at (a) $4.8 \mathrm{GHz}$, (b) $8.4 \mathrm{GHz}$, (c) $15.1 \mathrm{GHz}$, and (d) $22.2 \mathrm{GHz}$ with electric polarization vectors overlayed. Contour levels begin at $0.4 \mathrm{mJy}_{\text {beam }}{ }^{-1}$ in (a) and (b) and at $5.5 \mathrm{mJy} \mathrm{beam}^{-1}$ in (c) and (d) and increase by factors of 2. A vector length of 1 mas corresponds to a polarized flux density of (b) $0.42 \mathrm{mJy} \mathrm{beam}^{-1}$, (c) $0.83 \mathrm{mJy}_{\text {beam }}{ }^{-1}$, and (d) $1.7 \mathrm{mJy}_{\text {beam }}{ }^{-1}$. The bar in the lower right represents 5 mas or $\sim 45$ pc. 


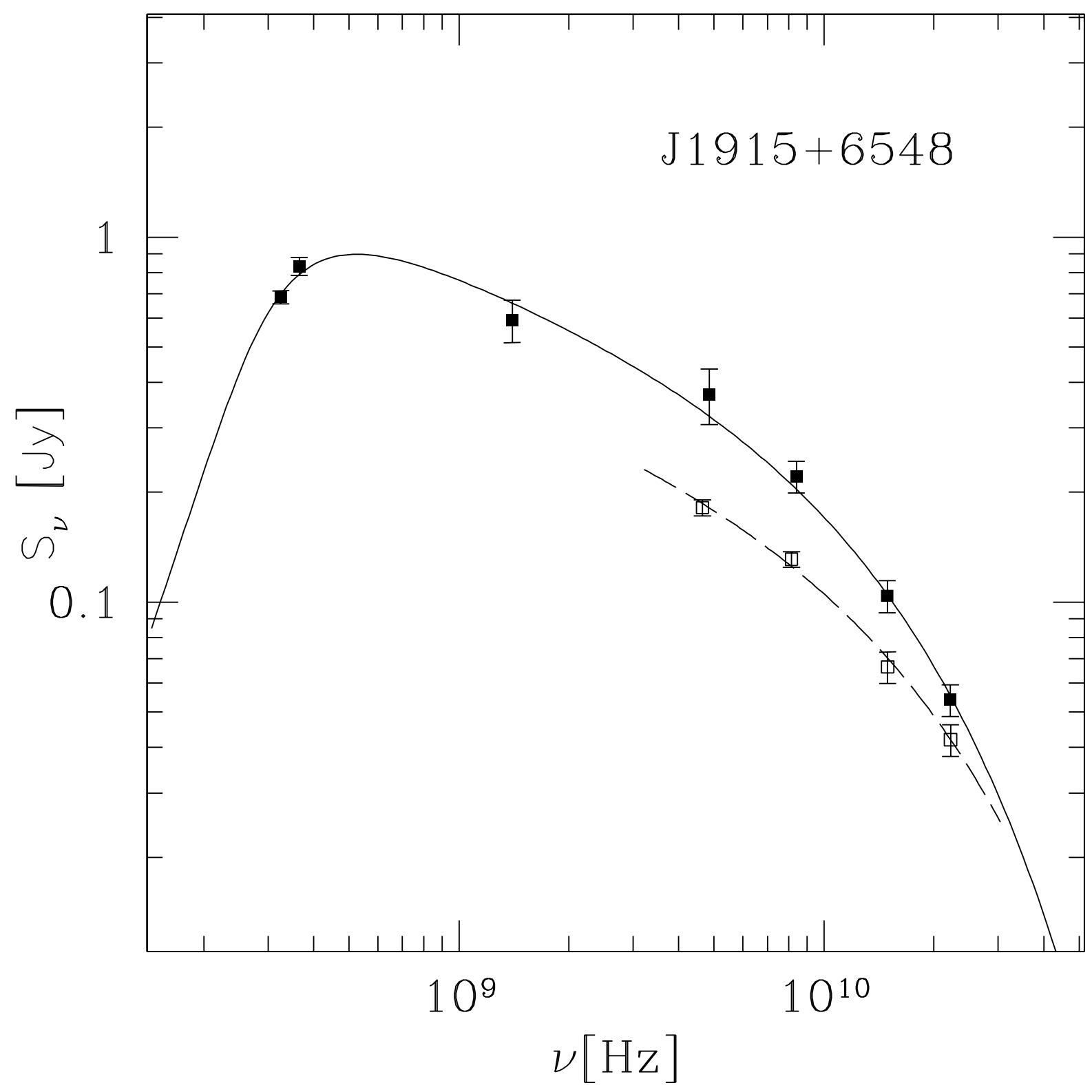

Fig. 6. - Total power spectrum of J1915+6548 using data points from the NASA Extragalactic Database and the data presented in this paper (filled squares). The break frequency is $17 \mathrm{GHz}$. Overlayed is the spectrum of component A which has a spectral index of -0.9 (open squares). 


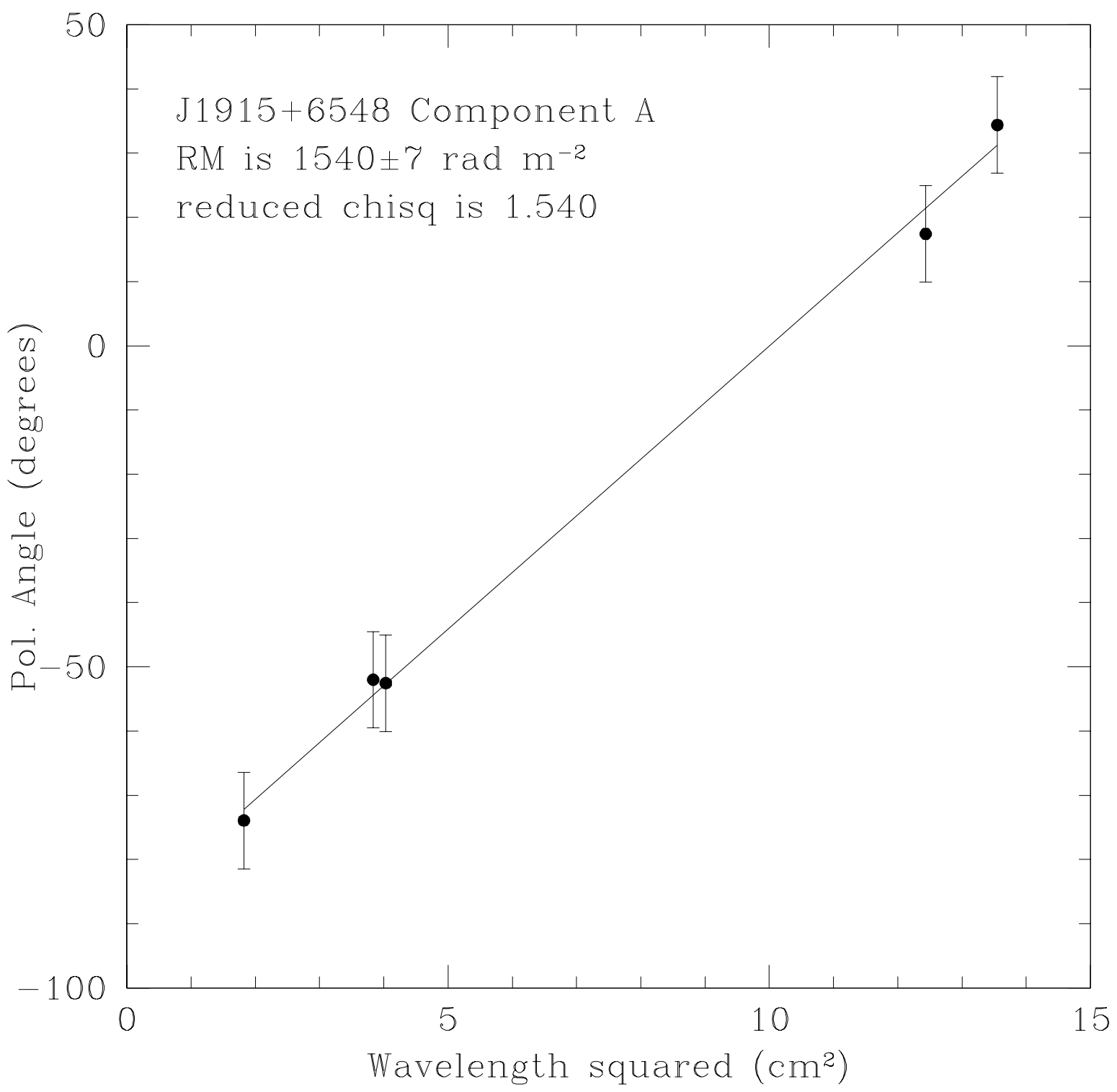

Fig. 7.- Plot of polarization angle versus wavelength squared for component A of $\mathrm{J} 1915+6548$ where the slope of the least squares line is the rotation measure. Note that $x$ and $y$ scales are different. 

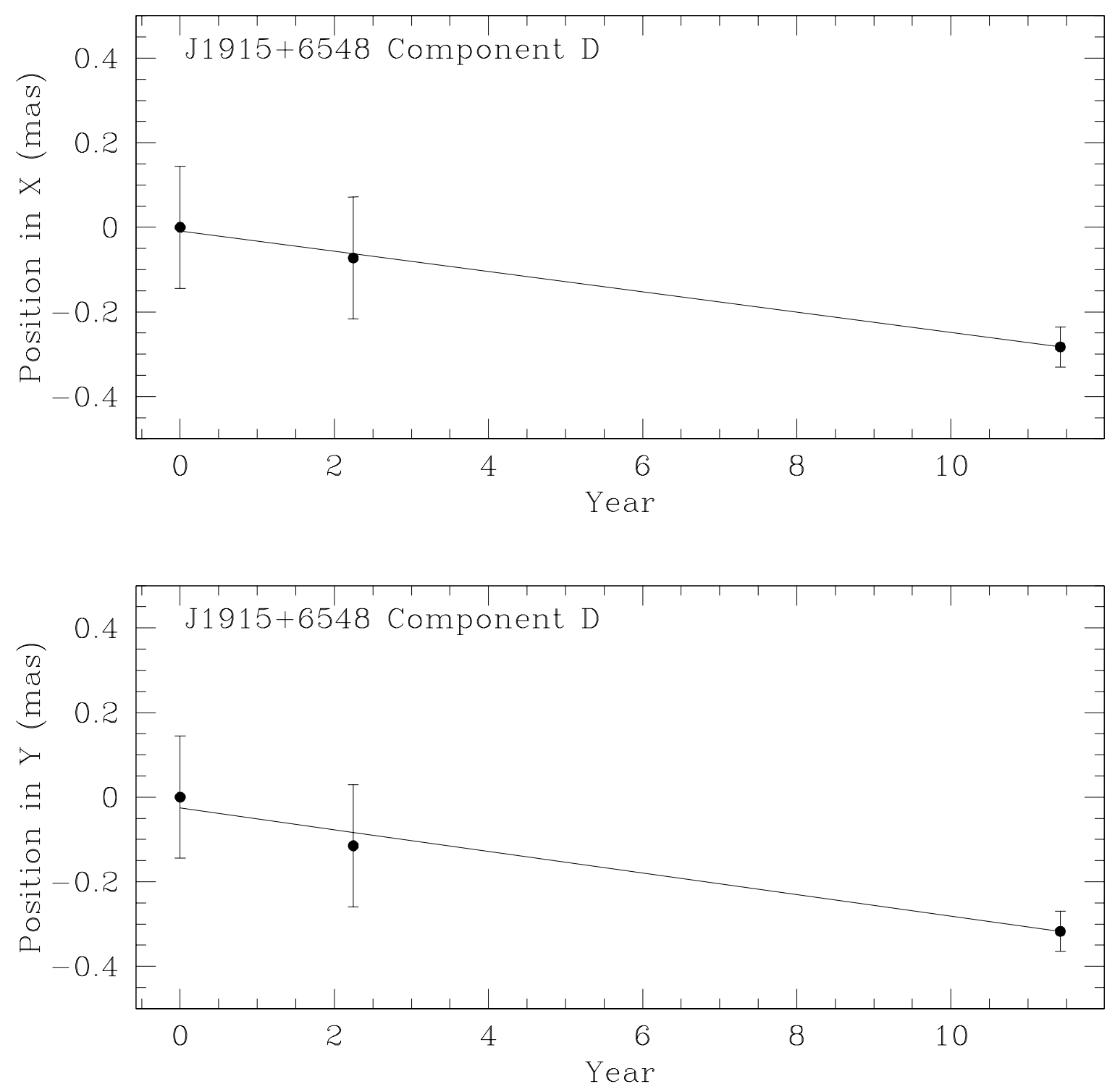

Fig. 8. - Proper motion plots of J1915+6548 with three epochs of model fit to the $4.8 \mathrm{GHz}$

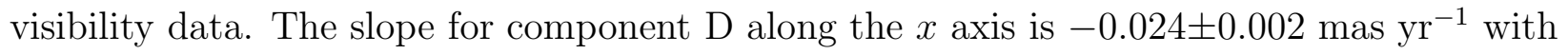

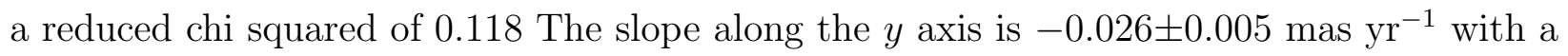
reduced chi squared of 1.153 . 

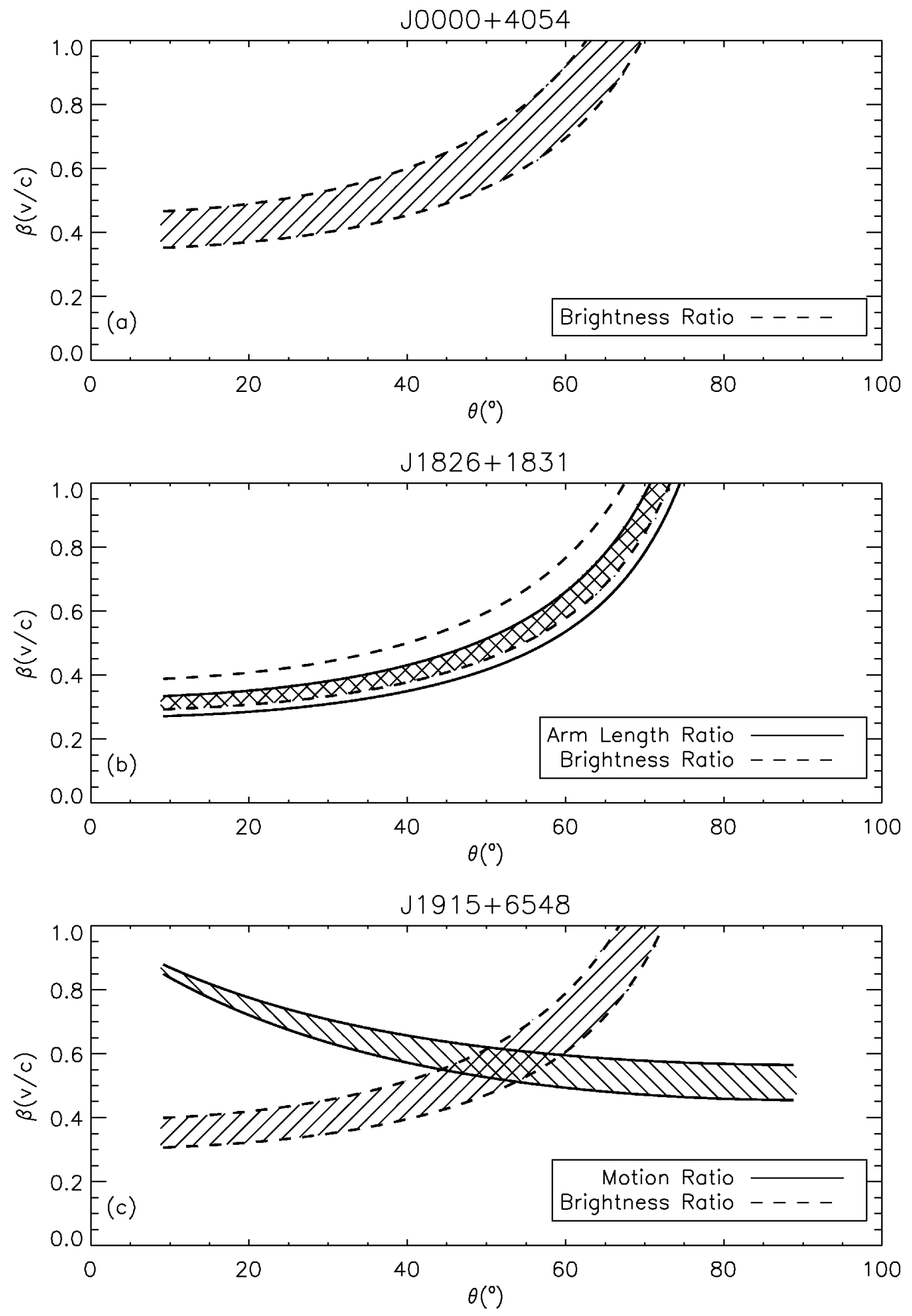

Fig. 9.- Plots of loci for intrinsic jet velocity, $\beta$, vs. angle to the line of sight, $\theta$, for (a) J0000+4054, (b) J1826+1831, and (c) J1915+46548. The brightness ratios are calculated from two different models with the hatching to indicate that the values lie between the lines. The arm length and motion ratios are hatched in between the upper and lower limits due to experimental error. Cross-hatching indicates where the regions overlap. Single-hatching is removed from (b) for clarity. 
Table 1. Observational Parameters

\begin{tabular}{cccccc}
\hline \hline Source & Date & $\begin{array}{c}\text { Freq. } \\
(\mathrm{GHz}) \\
(1)\end{array}$ & $\begin{array}{c}\text { Time } \\
(\mathrm{min}) \\
(4)\end{array}$ & $\begin{array}{c}\text { Bandwidth } \\
(\mathrm{MHz})\end{array}$ & $\begin{array}{c}\mathrm{rms} \\
(\mathrm{mJy}) \\
(6)\end{array}$ \\
\hline \multirow{2}{*}{$\mathrm{J} 0000+4054$} & 20050212 & 4.6 & 200 & 16 & 0.18 \\
& 20050212 & 5.0 & 200 & 16 & 0.12 \\
& 20050212 & 8.2 & 200 & 16 & 0.12 \\
& 20050212 & 8.5 & 200 & 16 & 0.09 \\
$\mathrm{~J} 1826+1831$ & 20050218 & 4.6 & 212 & 16 & 0.17 \\
& 20050218 & 5.0 & 212 & 16 & 0.18 \\
& 20050218 & 8.2 & 212 & 16 & 0.20 \\
& 20050218 & 8.5 & 212 & 16 & 0.19 \\
$\mathrm{~J} 1915+6548$ & 20041111 & 4.6 & 58 & 16 & 0.23 \\
& 20041111 & 5.0 & 58 & 16 & 0.18 \\
& 20041111 & 8.2 & 39 & 16 & 0.17 \\
& 20041111 & 8.5 & 39 & 16 & 0.15 \\
& 20041111 & 14.9 & 97 & 16 & 0.26 \\
& 20041111 & 15.3 & 97 & 16 & 0.21 \\
& 20041111 & 22.2 & 135 & 32 & 0.18 \\
\hline
\end{tabular}

*Notes - (1) J2000 source name; (2) Date of observation; (3) Frequency in GHz; (4) Integration time in minutes; (5) Bandwidth in MHz; (6) rms noise in mJy. Note that the rms for each fully averaged frequency, $4.8,8.4$, and $15.1 \mathrm{GHz}$, is generally a factor of $\sqrt{2}$ lower. 
Table 2. Source Parameters

\begin{tabular}{ccccccccccc}
\hline \hline $\begin{array}{c}\text { Name } \\
(1)\end{array}$ & $\begin{array}{c}\mathrm{RA} \\
(2)\end{array}$ & $\begin{array}{c}\mathrm{Dec} \\
(3)\end{array}$ & $\begin{array}{c}\mathrm{ID} \\
(4)\end{array}$ & $\begin{array}{c}M_{v} \\
(5)\end{array}$ & $\begin{array}{c}z \\
(6)\end{array}$ & $\begin{array}{c}S_{5 \mathrm{GHz}} \\
(7)\end{array}$ & $\begin{array}{c}S_{8 \mathrm{GHz}} \\
(8)\end{array}$ & $\begin{array}{c}S_{15 \mathrm{GHz}} \\
(9)\end{array}$ & $\begin{array}{c}S_{22 \mathrm{GHz}} \\
(10)\end{array}$ \\
\hline $\mathrm{J} 0000+4054$ & 000053.081551 & +405401.79335 & $\mathrm{G}$ & 21.4 & $\ldots$ & 521 & 322 & $\ldots$ & $\ldots$ \\
$\mathrm{J} 1826+1831$ & 182617.710882 & +183152.88973 & $\ldots$ & $\ldots$ & $\ldots$ & 427 & 279 & $\ldots$ & $\ldots$ \\
$\mathrm{J} 1915+6548$ & 191523.819114 & +654846.38505 & $\mathrm{G}$ & 18.2 & 0.486 & 331 & 202 & 104 & 54 \\
\hline
\end{tabular}

*Notes - (1) J2000 source name; (2) Right ascension and (3) Declination in J2000 coordinates from the VLBA Calibrator Survey by Beasley et al. 2002; (4) Optical host galaxy identification; (5) Optical magnitude; (6) Redshift; (7) Total flux density at $4.8 \mathrm{GHz}$ in mJy; (8) Total flux density at $8.4 \mathrm{GHz}$ in mJy; (9) Total flux density at $15.1 \mathrm{GHz}$ in mJy; (10) Total flux density at $22.2 \mathrm{GHz}$ in mJy. 
Table 3. CSO Model Parameters

\begin{tabular}{|c|c|c|c|c|c|c|c|c|c|}
\hline $\begin{array}{c}\text { Source } \\
(1)\end{array}$ & $\begin{array}{c}\text { Component } \\
\text { (2) }\end{array}$ & $\begin{array}{c}b_{m a j} \\
(\mathrm{mas}) \\
(3)\end{array}$ & $\begin{array}{c}b_{\min } \\
(\operatorname{mas}) \\
(4)\end{array}$ & $\begin{array}{c}\phi \\
\text { (deg.) } \\
(5)\end{array}$ & $\begin{array}{c}S \\
(\mathrm{mJy}) \\
(6)\end{array}$ & $\begin{array}{c}P \\
(\mathrm{mJy}) \\
(7)\end{array}$ & $\begin{array}{c}\mu \\
\left(\operatorname{mas~yr}^{-1}\right) \\
(8)\end{array}$ & $\begin{array}{l}v \\
(c) \\
(9)\end{array}$ & $\begin{array}{c}\text { Kinetic Age } \\
(\mathrm{yr}) \\
(10)\end{array}$ \\
\hline \multirow[t]{4}{*}{$\mathrm{J} 0000+4054$} & A & 2.28 & 1.71 & -3.4 & 14 & $<0.2$ & $<0.066$ & $\ldots$ & $>610$ \\
\hline & B1 & 0.91 & 0.42 & -83.6 & 19 & $<0.2$ & $\ldots$ & $\ldots$ & $\ldots$ \\
\hline & $\mathrm{B} 2$ & 0.63 & 0.63 & $\ldots$ & 28 & $<0.2$ & $\ldots$ & $\ldots$ & $\ldots$ \\
\hline & $\mathrm{C}$ & 3.01 & 2.41 & -65.4 & 205 & 2.1 & Reference & $\ldots$ & $\ldots$ \\
\hline \multirow[t]{4}{*}{$\mathrm{J} 1826+1831$} & $\mathrm{~A}$ & 8.05 & 4.51 & 24.6 & 12 & $<0.5$ & $\ldots$ & $\ldots$ & $\ldots$ \\
\hline & B & 0.45 & 0.45 & $\ldots$ & $11^{a}$ & $<0.5$ & Reference & $\ldots$ & $\ldots$ \\
\hline & $\mathrm{C}$ & 0.96 & 0.77 & 43.3 & 32 & 2.3 & $0.032 \pm 0.003$ & $\ldots$ & $450 \pm 43^{b}$ \\
\hline & $\mathrm{D}$ & 1.74 & 1.11 & -71.3 & 115 & $<0.5$ & $0.015 \pm 0.003$ & $\ldots$ & $2600 \pm 490$ \\
\hline \multirow[t]{4}{*}{$\mathrm{J} 1915+6548$} & $\mathrm{~A}$ & 0.46 & 0.32 & 25.2 & 183 & $<0.3$ & Reference & $\ldots$ & $\ldots$ \\
\hline & $\mathrm{B}$ & 1.88 & 0.86 & 29.8 & 72 & $<0.3$ & $\ldots$ & $\ldots$ & $\ldots$ \\
\hline & $\mathrm{C}$ & 1.65 & 1.37 & 24.9 & 36 & $<0.3$ & $\ldots$ & $\ldots$ & $\ldots$ \\
\hline & $\mathrm{D}$ & 1.30 & 0.88 & 17.6 & 15 & $<0.3$ & $0.035 \pm 0.004$ & $1.02 \pm 0.11$ & $940 \pm 110$ \\
\hline
\end{tabular}

*Notes - (1) J2000 source name; (2) Component name; (3) Major and (4) minor axes of Gaussian model component; (5) Position angle of major axis; (6) Integrated flux density of Gaussian model component; (7) Polarized intensity, or $3 \sigma$ limit; (8) Relative proper motion; (9) Relative proper motion in terms of $c$ if $z$ is available; (10) Kinematic age estimate. For J0000+4054 and J1826+1831, fluxes are at 8.4 GHz at 2005.118. For J1915+6548, fluxes are at $4.8 \mathrm{GHz}$ at 2004.863 .

${ }^{a}$ Correction: Gugliucci et al. (2005) erroneously listed the flux densities of component B as 80, 90 and $70 \mathrm{mJy}$ in epochs 1,2 , and 3 . The correct fluxes are 8, 9, and $7 \mathrm{mJy}$, respectively. This gives a core fraction of $3 \%$.

${ }^{\mathrm{b}}$ This is not a source age, but a kinematic age estimate for a jet component. 
Table 4. Hotspot Ratios for Polarized and Unpolarized CSOs

\begin{tabular}{|c|c|c|c|c|c|}
\hline (1) & $\begin{array}{c}\text { Source } \\
(2)\end{array}$ & $\begin{array}{c}S_{1} \\
(\mathrm{mJy}) \\
(3)\end{array}$ & $\begin{array}{c}S_{2} \\
(\mathrm{mJy}) \\
(4)\end{array}$ & $\begin{array}{c}\text { Ratio } \\
\left(\frac{S_{2}}{S_{1}}\right) \\
(5)\end{array}$ & $\begin{array}{c}\text { Core } \\
\text { Fraction } \\
(6)\end{array}$ \\
\hline \multirow[t]{3}{*}{ Polarized Sources } & $\mathrm{J} 0000+4054$ & 205 & 14 & 0.07 & $<0.0006$ \\
\hline & $\mathrm{J} 1826+1831$ & 115 & 12 & 0.10 & 0.04 \\
\hline & $\mathrm{J} 1915+6548$ & 153 & 6 & 0.04 & $<0.001$ \\
\hline \multirow[t]{12}{*}{ Unpolarized Sources } & $\mathrm{J} 0003+4807$ & 38 & 13 & 0.34 & 0.04 \\
\hline & J0204+0903 & 120 & 68 & 0.57 & 0.20 \\
\hline & $\mathrm{J} 0427+4133$ & 65 & 28 & 0.43 & 0.86 \\
\hline & $\mathrm{J} 0620+2102$ & 156 & 88 & 0.56 & $<0.001$ \\
\hline & J0754+5324 & 39 & 36 & 0.92 & $<0.003$ \\
\hline & $\mathrm{J} 1111+1955$ & 98 & 76 & 0.78 & $<0.002$ \\
\hline & $\mathrm{J} 1143+1834$ & 130 & 99 & 0.76 & $<0.001$ \\
\hline & $\mathrm{J} 1414+4554$ & 64 & 51 & 0.80 & $<0.003$ \\
\hline & $\mathrm{J} 1546+0026$ & 213 & 110 & 0.52 & 0.40 \\
\hline & $\mathrm{J} 1734+0926$ & 183 & 119 & 0.65 & $<0.0006$ \\
\hline & $\mathrm{J} 1816+3457$ & 135 & 59 & 0.44 & $<0.001$ \\
\hline & $\mathrm{J} 2203+1007$ & 145 & 53 & 0.37 & $<0.001$ \\
\hline
\end{tabular}

*Notes - (1) Category; (2) J2000 source name; (3) Integrated flux density of Gaussian model component of brighter hotspot at $8.4 \mathrm{GHz}$; (4) Integrated flux density of Gaussian model component of dimmer hotspot at $8.4 \mathrm{GHz}$; (5) Ratio of dimmer hotspot as compared to brighter hotspot; (6) Fraction of total flux that is attributed to the core (using $3 \sigma$ limit for core flux if not detected). Fluxes for unpolarized CSOs from 2000.227 in GTPG. Fluxes for polarized CSOs and candidate from this paper. 